\title{
PredicTF: a tool to predict bacterial transcription factors in complex
} microbial communities.

3

4 Lummy Maria Oliveira Monteiro ${ }^{1,2,3}$, Joao Saraiva ${ }^{1}$, Rodolfo Brizola Toscan ${ }^{1}$, Peter F Stadler ${ }^{2}$, Rafael Silva-Rocha ${ }^{3}$, Ulisses Nunes da Rocha ${ }^{1 *}$

6

$7 \quad{ }^{1}$ Helmholtz Center for Environmental Research (UFZ), Leipzig, Germany

$8{ }^{2}$ Bioinformatics Group, Institute of Computer Science, Universität Leipzig, Leipzig, Germany

$9 \quad{ }^{3}$ Ribeirão Preto Medical School (FMRP), University of São Paulo (USP), Ribeirão Preto, Brazil 10

11

12 *Correspondence: Ulisses Nunes da Rocha, ulisses.rocha@ufz.de

13

14 Authors' email addresses:

15 Lummy Maria Oliveira Monteiro - $\underline{\text { lummymaria@gmail.com }}$

16 João Saraiva - joao.saraiva@ufz.de

17 Rodolfo Brizola Toscan - rodolfo.toscan@ufz.de

18 Peter F. Stadler - studla@bioinf.uni-leipzig.de

19 Rafael Silva-Rocha - silvarochar@usp.br

20 Ulisses Nunes da Rocha - ulisses.rocha@ufz.de 


\section{Abstract}

28 Transcription Factors (TFs) are proteins that control the flow of genetic information by

29 regulating cellular gene expression. Here we describe PredicTF, a first platform

30 supporting the prediction and classification of novel bacterial TF in complex microbial

31 communities. We evaluated PredicTF using a two-step approach. First, we tested

32 PredictTF's ability to predict TFs for the genome of an environmental isolate. In the

33 second evaluation step, PredicTF was used to predict TFs in a metagenome and 11

34 metatranscriptomes recovered from a community performing anaerobic ammonium

35 oxidation (anammox) in a bioreactor. PredicTF is open source pipeline available at

36 https://github.com/mdsufz/PredicTF.

Keywords: Gene regulation, Transcription factors, Deep Learning, Transcription factor database, Microbial Communities

\section{Background}

The functional potential of microbial communities can be determined by the genetic content of its constituent members. However, genetic content alone does not guarantee that a given function or enzymatic reaction will be performed [1]. In this scenario, Transcription Factor proteins (TFs) play a central and critical role in gene regulation. These proteins are responsible for optimizing proteins and structural RNAs and the subsequent levels of metabolites and other properties, ensuring the survival and adaptation of organisms to the most diverse types of stress and environmental changes

49 [2]. The activity of bacterial TFs is modulated by environmental signals (e.g. changes in the oxygen condition, temperature, $\mathrm{pH}$ or the lack of a specific substrate) [3].

51 Additionally, for many promoters, combinations of transcription factors work together 
to integrate different signals $[2,4]$. TFs can also work with other DNA-binding proteins whose primary role is to sculpt the bacterial folded chromosome [2,5]. Knowledge of the TFs profile expressed by an organism is the first step to better understand the regulatory network that controls protein expression in an organism or a community. Since TFs may determine when and which genes are expressed, profiling TFs can help understand the regulation of gene expression and to build regulatory networks in complex microbial communities. Further, defining which factors control gene expression may offer insights into the mechanisms controlling ecosystem processes and even interactions between species of a microbial community. However, current TF databases are focused on single or small groups of genomes. These databases are largely manually curated based on literature evidence and pairwise sequence comparison of genomes from model organisms. Examples of these databases include RegulonDB for Escherichia coli K-12 [6], DBTBS for Bacillus subtilis [7], FlyBase for Drosophila [8], and FTFD for fungal species [9]. DBD [10], is a database generated from the prediction of TFs from 150 sequenced genomes from across the tree of life. Unfortunately, DBD has not been updated for more than 9 years.

One of the major goals in the manipulation of microbiomes for ecological and biotechnological applications is to control the outcome of their functions [11]. As TFs are key to potentially control which genes are expressed, one of the best ways to study and understand gene regulation in a microbiome may be to profile its TFs. To date, no platform supports prediction and classification of novel bacterial TF from 'omics data recovered from microbial communities.

Deep Learning approaches have been used to predict DNA sequence affinities [12] and to identify TF-binding sites in humans [13]. Although deep learning has been used in gene regulation, it has never been used to predict bacterial TFs. Further, the need for a user-friendly tool for prediction of TFs that could assist in gene regulation 
82

analysis motivated the development of PredicTF. PredicTF is a deep learning tool used to predict and identify TFs from full protein-length sequences. Further, we constructed a robust database for bacterial transcriptional factors (BacTFDB) that was used to train our Deep Learning model.

\section{Results and Discussion}

PredictTF is a command line software for prediction of novel transcription factors from genomic and metagenomic data. We created a bacterial transcription factor database (BacTFDB) by merging and manually curating TFs present in CollectTF [14] and the Universal Protein Resource (UniProt) [15]. CollectTF provides well described and characterized, in vivo validated, TFs while UniProt is a comprehensive resource for protein sequence and annotation data. We used BacTFDB to train a deep learning model to predict new TFs and their families in genomes and metagenomes. Five model organisms (Escherichia coli, Bacillus subtillis, Pseudomonas fluorescens, Azotobacter vinelandii and Caulobacter crescentus) were used to test the performance and accuracy of PredicTF. We used the same approach to predict TFs from a clinical isolate $(P$. aeruginosa PAO1) and a metagenome sample isolated from an anaerobic ammonium oxidation community. We also determined if the predicted TFs were expressed in transcriptomes (isolate) and metatranscriptomes (microbial community), respectively (Fig. 1).

\section{Database}

BacTFDB is a robust and versatile bacterial TF database, it contains 11.691 TFs amino acid sequences spanning 1049 TF families and 720 different bacterial species. Fig. 2 shows the database distribution based on TF families and regulatory elements 
103 (Fig. 2A) and the distribution based on bacterial species (Fig. 2B). Although BacTFDB

104 is composed by 11.961 TFs elements from 1049 different families and 720 organism's

105 species, Fig. 2 shows TFs families and species that accumulate more than 50 sequences.

106 We will update BacTFDB annually by adding novel entries deposited in UniProt and

107 CollecTF. BacTFDB was used in PredicTF’s deep learning model training. This model

108 was later used to predict new TFs and their families in genomes and metagenomes.

Performance and Accuracy

The performance and accuracy of PredicTF were evaluated through the

112 prediction of TFs in five model organisms (E. coli, B. subtillis, P. fluorescens, $A$.

113 vinelandii and C. crescentus). For each model organism a different PredicTF model was

114 trained to predict TFs from full protein-length sequences (described in the

115 implementation section).

116 The performance of PredicTF to identify TFs in the different model organisms

117 ranged from $27 \%$ to $60 \%$ of the proteins described as TFs in the genomes of model

118 organisms and the accuracy for experimentally validated TFs ranged from $73.91 \%$ and

119 91.43\% (Table 1). Further, PredicTF was able to identify putative annotated TFs in the

120 genomes of E. coli and B. subtillis with accuracies $85.71 \%$ and $100 \%$, respectively

121 (Table 1). No novel TF was predicted in the genome of $C$. crescentus, $P$. fluorescens

122 and A.vinelandii (Table 1). TFs predicted by PredicTF for each organism, sorted by TF

123 family, are shown in Fig. 3. For all organisms tested the most predicted TF family was

124 LysR followed by OmpR/PhoB. The degree of accuracy obtained by PredicTF suggests

125 that the deep learning strategy used is promising for the prediction of TFs in genomic or

126 metagenomic data of bacterial species. PredicTF performance and accuracy can be

127 further improved by expanding the number and diversity of sequences present in 
128 BacTFDB. As BacTFDB will be update yearly, we expect an improvement in TF

129 identification of with every update.

131 Table 1. PredicTF performance, accuracy for experimentally validated Transcription

132 Factors (Accuracy EV) and accuracy for putative Transcription Factors (Accuracy PU)

133 in genomes of model organisms.

\begin{tabular}{cccc}
\hline Organism & Performance $^{\mathbf{a}}$ & Accuracy EV $^{\mathbf{b}}$ & Accuracy PU $^{\mathbf{c}}$ \\
\hline E. coli $\mathbf{k 1 2}$ & $35.40 \%$ & $88.51 \%$ & $85.71 \%$ \\
B. subtillis & $27.23 \%$ & $73.91 \%$ & $100 \%$ \\
C. crescentus & $38.04 \%$ & $83.93 \%$ & - \\
P. fluorescens & $51.19 \%$ & $91.43 \%$ & - \\
A.vinelandii & $60.53 \%$ & $90.40 \%$ & - \\
\hline
\end{tabular}

a Performance was calculated by the ratio of the total number of TFs predicted by PredicTF (Predicted TFs) to the total number of proteins annotated as TFs in NCBI (Annotated TFs) multiplied by 100;

${ }^{\mathrm{b}}$ Accuracy EV was determined by the ratio of the total number of TFs predicted by PredicTF in agreement with NCBI annotation (TFs predicted correctly) to the total number of TFs predicted by PredicTF (TFs predicted) multiplied by 100;

${ }^{c}$ Accuracy TU was determined by the total number of putative TFs predicted correctly divided by putative TFs predicted multiplied by 100; Putative TFs predicted correctly is the total number of putative TFs predicted correctly by PredicTF in agreement with NCBI annotation; and, Putative TFs predicted is the total number of putative TFs predicted by PredicTF;

${ }^{\mathrm{d}}$ Currently there are no putative annotated TFs described in the genome of $C$. crescentus, $P$. fluorescens and A.vinelandii

Mining and Predicting TFs in Genomes and Transcriptomes from a bacterial

\section{isolate using PredicTF}

PredicTF was used to predict TFs on the genome of P. aeruginosa PAO1 and

146 these TFs were mapped in transcriptomes from the same isolate [16]. PredicTF

147 predicted a total of 199 TFs in the P. aeruginosa PAO1 genome shown in Additional

148 file 1: Fig. S1A by a family’s distribution graphic. These 199 TFs were mapped in the

149 transcriptomic data of a reference of P. aeruginosa PAO1. Initially, the mapping was

150 done in the transcriptome of P. aeruginosa PAO1 cultured in LB media. Using this

151 strategy, we were able to map 69 of the 199 predicted TFs to the transcriptomes under

152 the experimental conditions carried out by Hwang \& Yoon, 2019 (Additional file 1: Fig.

153 S1B) [16]. Next, the mappings were done for another three clinical mutants of $P$. 
each $P$. aeruginosa PAO1 mutant cultured in presence of antibiotic cocktail is shown in the supplementary data (Additional file 2: Fig. S2B, S2D and S2F). These results environmental conditions.

\section{Mining and Predicting TFs in a Metagenome and Metatranscriptome using}

PredicTF

PredicTF was used to profile TFs in one metagenome recovered from an anaerobic ammonium oxidation community [17] followed by the mapping of the predicted TFs in metatranscriptomes recovered from the same community

167 (metatranscriptomes accession numbers can be found in Additional file 3: Table S1). A total of 792 TFs (Fig. 4A) were predicted in LAC_MetaG_1, an anaerobic ammonium oxidizing microbial community from an anammox membrane bioreactor [17]. These 792 TFs are distributed across 27 TF families (Fig. 4A) and are related to the regulation

171 of functions such as the oxygen limitation response and late symbiotic functions

172 (NarL/FixJ), phosphate regulon response (OmpR/PhoB), transcriptional activator for nitrogen-regulated promoters (NtrC/DctD) and ferric uptake regulation (Fur). To determine how a traditional annotation pipeline identify potential TF we used Prokka

175 [18]. This tool was able to identify 1815 ORFs (Additional file 4: Table S2). PredicTF

176 can be used with no previous knowledge regarding transcription factors, it is fast and it

177 requires low memory when compared to Blast based annotation and it indicates only

178 results of TFs with a specific TF family annotation. On the other hand, to identify TFs

179 using Prokka one would need specialized training to mine the general annotation.

180 Therefore, scientists with general microbiology background may take a long time to

181 undergo this task. Further, Prokka gives no indication to the TF families of the putative 
182 annotated TFs. Time is also a drawback of using Prokka to mine TFs, we calculated we

183 needed over $400 \mathrm{~h}$ to perform mine one single metagenomics library; in comparison,

184 PredicTF needed $2 \mathrm{~h}$ to identify TF in the same metagenomics library.

185 Next, the 792 TFs were mapped in 11 metatranscriptomes collected in different

186 dates from the same bioreactor where the metagenome was recovered (Additional file 5:

187 Table S3, Fig. 4B). Clustering analysis demonstrated the presence of five different

188 groups of TFs families based on the number of transcription factor families expressed in

189 each library (Fig. 4B). It is interesting to note that the two most abundant clusters in the

190 heatmap are directly related to the oxygen limitation caused by the anaerobic

191 ammonium oxidizing cultivation. In a bioreactor where oxygen is limited, an increase in

192 the amount of nitrogen and phosphate is expected. The presence of $\mathrm{N}$ and $\mathrm{P}$ diverts the

193 metabolism of the microbial community towards the production of regulators (TFs) that

194 help to maintain community stability. Clustering analyzes can be helpful to demonstrate

195 the similarity between metatranscriptomic libraries based on the occurrence of TFs. This

196 strategy can be useful to compare the profiles of TFs expressed in different

197 environmental situations (comparing libraries with different metadata) creating patterns

198 of TFs expression. Exploration of TF profiling in microbial communities (metagenomes

199 or metatranscriptomes) will allow the exploration of regulation within complex

200 microbial communities. Further, The recovery of metagenome assembled genomes is

201 becoming standard in metagenomics studies [19-21]. The use of PredicTF together with

202 the recovery of metagenome assembled genomes will allow the exploration of species-

203 specific molecular mechanisms involved in the regulation of different ecosystem

204 processes.

205

206 Conclusions 
A better understanding of TFs in a bacterial community context open revenue

208 for the exploration of gene regulation in ecosystems where bacteria play a key role. Our

209 deep learning strategy was based on a novel and robust TF bacterial database

210 (BacTFDB) with over 11 thousand TFs and their respective families. BacTFDB is a

211 unique resource for the exploration of TFs and it provided the data to train a model

212 within PredicTF capable of predicting novel TFs from genomes and metagenomes.

213 PredicTF is the first pipeline designed to predict and annotate TFs in complex microbial

214 communities. The prediction of TFs can provide information for those aiming to study

215 and understand bacterial communities within a context of gene regulation. We also

216 demonstrated that PredicTF can be used to predict novel TFs in metagenomes and

217 metatrascriptomes creating the potential profile for regulatory elements in complex

218 microbial communities.

219 PredicTF is a flexible open source pipeline able to predict and annotate TFs in

220 genomes and metagenomes and can be found at https://github.com/mdsufz/PredicTF.

\section{Methods}

\section{BacTFDB - Bacterial Transcription Factor Data Base}

To create a novel Bacterial Transcription Factor Data Base (BacTFDB), we collected data from two publicly available databases. Initially, we chose to collect data from CollecTF [14], a well described and characterized database. Since CollecTF does not provide an application programming interface (API) for bulk download, we developed a Python code (version 2.7) using the Beautiful Soup 4.4.0 library to recover the data from CollecTF. With this strategy we listed 390 TF experimentally validated

230 amino acid sequences distributed over $44 \mathrm{TF}$ families. The script can be found at 
233 UniProt's API. We downloaded sequences of interest by adding a filter with the key

(Swiss-Prot) - Manually annotated sequences that belonged to the bacteria taxonomy.

The UniProt API was accessed on $8^{\text {th }}$ September-2019 and a total of 21.581 TF amino

acid sequences, with applied filters, were collected. We merged the data collected from

CollecTF and UniProt databases which resulted in a total of 21.971 TFs. Next, we

was designed to also assign TF family. Finally, a manual inspection was performed to

242 remove case sensitive and presence of characters associated to the database header. The

243 first version of BacTFDB contains a total of 11.691 unique TF sequences. A summary

244 of the information contained in BacTFDB can be found in the supplementary data

245 (Additional file 6: Fig. S4). To evaluate PredicTF in model organisms we created 5

246 subsets of BacTFDB. The description of these subsets can be found in the

247 supplementary data (Additional file 7: Table S4).

\section{Mapping Transcription Factors using PredictTF}

We used a deep learning approach similar to that found in DeepARG [22].

Supervised machine learning models are usually divided into characterization, training, and prediction units. Briefly, our approach uses the concept of dissimilarity-based

253 classification [23] where sequences are represented and featured by their sequence

254 similarity to known genes. BacTFDB was used to train and test the deep learning model

255 (https://github.com/mdsufz/PredicTF) and latter validated in model organisms. Next,

256 PredicTF was used to predict novel TFs from full protein-length sequences in genomes 
257

258

259

260

261

262

263

264

265

266

267

268

269

270

271

272

273

274

275

276

277

278

279

280

281

and in one metagenome. After prediction, the data was mapped in transcriptomes and metatranscriptomes from samples where the genetic potential was determined.

Using PredicTF, we trained five different models - one for each model organism (Additional file 3: Table S1). For each model, the TFs affiliated with the respective model organism were removed prior to training to avoid overfitting. PredicTF-no-coli was trained to predict TFs in E. coli, PredicTF-no-subtilis was trained to predict TFs in B. subtilis, PredicTF-no-crescentus was trained to predict TFs in C. crescentus, PredicTF-no-fluorescens was trained to predict TFs in P. fluorescens and PredicTF-novinelandii was trained to predict TFs in A. vinelandii.

\section{Performance and accuracy calculation}

We evaluated PredicTF by calculating accuracy and performance. Performance can be deemed to be the fulfillment of a task. In PredicTF case, performance is how good TF predictions are. Using model organisms (see later in the session Prediction of Transcription Factors in model organisms), performance was calculated by quantifying the number of TFs that PredicTF was able to predict divided by number of TFs already described and annotated for our model organisms (Additional file 7: Equation 1).

Accuracy indicates how correct the predictions performed by PredicTF are. Also using data of model organism, accuracy was determined by calculating the number of TFs correctly predicted divided by the total number of TFs predicted by PredicTF. We divided accuracy in two categories. In the first accuracy category, we determined accuracy against experimentally validated TFs (Additional file 7: Equation 2). In the second accuracy category, we determined accuracy against TFs without experimental validation (Additional file 7: Equation 3); i.e., putative TFs. The performance, accuracy, and accuracy for putative TFs were calculated as the ratio of predicted to annotated TFs. 
282 Accuracy was quantified as the fraction of correctly predicted TFs among all

283 predictions.

284

\section{Prediction of Transcription Factors in model organisms}

We selected bacterial species that have been widely studied as model organisms.

287 Some bacterial species became model organisms for TF studies because they are easy to

maintain and grow in a laboratory setting and to manipulate in pure culture experiments.

Five complete genomes from model organisms (E. coli, B. subtillis, P. fluorescens, A.

vinelandii and C. crescentus) were downloaded directly from NCBI. The strains details

and accession number (RefSeq) for all selected organisms are listed in the

supplementary data (Additional file 3: Table S1). By evaluating PredicTF using model

organisms (Additional file 6: Table S3) we extrapolated performance and accuracy of

our deep learn model. Since known TFs for each organism were removed from each the

training dataset, we eliminate the possibility of mapping TFs already known and

annotated for each of the different species. Performance, accuracy and accuracy for

putative TFs of PredicTF for these five model organisms were calculated using

298 Equations 1, 2 and 3.

299

\section{Prediction of Transcription Factors in a clinical isolate}

We demonstrated the use of PredicTF in a previously sequenced $P$. aeruginosa

302 (PAO1) genome, a clinical isolate publicly available in NCBI (accession number

303 NC_002516.2). P. aeruginosa PAO1 was selected because its genome has been sequenced and because of the availability of transcriptomes from three clinical mutants of PAO1 (Y71, Y82, and Y89) grown in the presence and absence of an antibiotic 
aeruginosa PAO1 mutants were isolated from the sputa of three different pneumonia

309 patients. Transcriptomes of P. aeruginosa PAO1 wild type and its mutants cultured in

310 two different conditions (LB medium and LB medium in presence of antibiotic cocktail)

311 have been previously described [16]. We used this data to determine the TF profile in

312 these P. aeruginosa PAO1 mutants grown in two different conditions.

313 PredicTF was first used to predict TFs in the P. aeruginosa PAO1 genome.

314 Next, the predicted TFs were mapped to the transcriptomes of the P. aeruginosa PAO1

315 mutants Y71, Y82 and Y89 (see later). Further description of the mapping of the

316 transcriptomes to the genomes is available at https://github.com/mdsufz/PredicTF. The

317 PredicTF model used in this step was trained with the full database BacTFDB. All

318 accession numbers used in this work are listed in the supplementary data (Additional

319 file 3: Table S1).

320

\section{Prediction of Transcription Factors in Complex Microbial Communities}

To test PredicTF in a complex microbial community, we used an anaerobic

bioreactor metagenome (LAC_MetaG_1) (data publicly available at NCBI bioproject

325 via accession number PRJNA511011) [17]. We removed short and low-quality reads using Trim Galore - v0.0.4 dev according developer’s instructions [24]. Over 50 million

reads survived this step and were assembled using the de novo assembler metaSPADES

- v3.12.0 [25]. The assembly was translated from nucleotide to amino acid sequences, assembly was then used as input for the prediction of transcription factors using

331 PredicTF. The region from each predicted TF was extracted. These putative TFs were later used in the mapping TFs to metatranscriptomes. 
We checked if the putative TFs predicted in the metagenomes were transcribed

334 by checking if the metatranscriptomic libraries were mapping to those regions. The

335 metatranscriptomic and metagenomic libraries used in this step belonged to the same

336 bioreactor. These metatranscriptomes are publicly available at the European Nucleotide

337 Archive under the accession numbers SRR7091385, SRR7523233, SRR7523244,

338 SRR7523245, SRR7091400, SRR7091401, SRR7091381, SRR7091402, SRR7091406,

339 SRR7523243, SRR7523246. These 11 metatranscriptomes were used to demonstrate the

340 effectiveness of the pipeline and to indicate the potential of PredicTF to profile

341 transcription factors in complex microbial communities. All accession numbers used in

342 this work are listed in the supplementary data (Additional file 3: Table S1).

343 To have a baseline comparison with a traditional annotation pipeline, we used

344 Prokka [18] to annotate the same anammox membrane bioreactor metagenome

345 (LAC_MetaG_1). We mined the annotation by hand with specialized knowledge of

346 scientists specialized in Transcription Factors. We did not determine the families as this

347 work would need to be done for every single hit individually using the output of Prokka.

\section{Mapping transcription factors to transcriptomes and metatranscriptomes}

Each transcriptomic and metatranscriptomic library was quality controlled by removing short and low-quality reads using Trim Galore - v0.0.4 dev [24]. The 7

transcriptomic libraries for the $P$. aeruginosa PAO1 wild type and mutants showed at

libraries yielded over 50 million reads per library after quality check. After, the

remaining transcriptomic and metatranscriptomic reads were mapped to their respective assembled genome or metagenome using Bowtie2 - v2.3.0 [27]. The number of reads 
transcriptomic or metatranscriptomic reads were then crossed-referenced with the

360 regions of their respective assembly which PredicTF assigned as putative TFs creating a

361 TF profile for each transcript and metatranscriptome. A detailed description on the mapping of RNA-seq data to their respective genome or metagenome assembly can be found at the PredicTF github (https://github.com/mdsufz/PredicTF).

364

365

List of Abbreviations

366

Transcription Factors (TFs)

367

Bacterial Transcription Factor Data Base (BacTFDB)

368

Transcription factor binding sites (TFBSs)

369

anaerobic ammonium oxidizing (anammox)

370

371

Declaration Sections

372

Ethics approval and consent to participate

373

Not applicable

Consent for publication

Not applicable

Availability of data and materials

379 Project name: PredicTF

380 Project home page: https://github.com/mdsufz/PredicTF

381 Operating system: Linux64

382 Programming languages: Python 2.7 
385 (http://deeplearning.net/software/theano/) [32]. Trim Galore - v0.0.4 dev

386 (https://www.bioinformatics.babraham.ac.uk/projects/trim_galore/) [24]. MetaSPADES

387 - v3.12.0 (https://github.com/ablab/spades\#meta) [25]. Emboss transeq

388 (http://www.bioinformatics.nl/cgi-bin/emboss/transeq) [26]. Bowtie2 - v2.3.0

389 (https://sourceforge.net/projects/bowtie-bio/) [27]. SAMTools - v1.9

390 (http://github.com/samtools/) [28].

391 Genomes of the model organisms used in the Tool Validation step are available

392 at the National Center for Biotechnology Information (https://www.ncbi.nlm.nih.gov/)

393 under the accession numbers NC_000913.3, NC_000964.3, NC_011916.1,

394 NC_021149.1, and NC_016830. The datasets supporting the Prediction of Transcription

395 Factors in a clinical isolate of this article are available at National Center for

396 Biotechnology Information (https://www.ncbi.nlm.nih.gov/) under the accession

397 number NC_002516.2 (genome) and study accession PRJNA479711 (transcriptomes).

398 The datasets used for the Prediction of Transcription Factors in Complex Microbial

399 Communities of this study are available at National Center for Biotechnology

400 Information (https://www.ncbi.nlm.nih.gov/) under the study accession PRJNA511011.

401 The respective data sets of metatranscriptomes used are available at National Center for

402 Biotechnology Information (https://www.ncbi.nlm.nih.gov/) under the SRA numbers

403 SRR7091385, SRR7523233, SRR7523244, SRR7523245, SRR7091400, SRR7091401,

404 SRR7091381, SRR7091402, SRR7091406, SRR7523243, SRR7523246 and the Joint

405 Genome Institute (https://jgi.doe.gov/) under the Gold Analysis Project identifiers

406 Gp0267156, Gp0267150, Gp0267154, Gp0267155, Gp0267157, Gp0267158,

407 Gp026715, Gp0267159, Gp0267152, Gp0267153, Gp0267160. All analysis, results and

408 scripts used to generate figures are available at https://github.com/mdsufz/PredicTF. 
411 Not applicable

412

413 Funding

414 LMOM were supported by FAPESP PhD (award \# 2016/19179-9) and FAPESP

415 Research Internship Scholarship Abroad (award \# 2018/21133-2). RSR was supported

416 by FAPESP (award \# 2019/15675-0). JS and UNR were supported by the Helmholtz

417 Young Investigator grant VH-NG-1248 Micro ‘Big Data’.

418

\section{Authors' contributions}

420 LMOM, PFS, RSR, and UNR developed the concept of PredicTF. LMOM, JS, UNR

421 developed the PredicTF workflow. LMOM, JS, and UNR performed the benchmarks.

422 LMOM provided information and data for the creation BacTFDB dataset. RBT and

423 UNR performed the metagenome and metatranscriptome analysis. LMOM and UNR

424 wrote the manuscript. All authors read and approved the manuscript.

425

426

Acknowledgements

427

428

429

430

431

432

433

434

435

436 


\section{Figure legends:}

438

$439 \quad$ Fig. 1

440 PredicTF workflow and testing. We collected publicly available data on TFs from two

441 different databases: CollecTF and UNIPROT. After removing redundancies and

442 filtering TFs well characterized, this data (BacTFDB) was used to train a deep learning

443 model to predict new TFs and their families. Five model organisms (Escherichia coli,

444 Bacillus subtillis, Pseudomonas fluorescens, Azotobacter vinelandii and Caulobacter

crescentus) were used to test the accuracy of PredicTF. Later, we used the same

approach to predict TFs from an isolate ( $P$. aeruginosa) and mapped TFs predicted in

447 transcriptomics data (P. aeruginosa and mutants in two experimental conditions).

448 Finally, we used our tool to predict TF for complex communities (metagenome) and mapped these TFs in their respective meta-transcriptomes.

Fig. 2

Database composition: Transcription Factor Database (BacTFDB) distribution. A)

453 Database distribution based on the TFs and B) Regulatory Elements families and

454 Organisms species. In these graphics only families with up to 50 sequences and only organisms that contributed with more than 50 sequences are shown.

$457 \quad$ Fig. 3

458 Prediction of TFs by PredicTF for genomes of model organisms. Prediction of TFs or 5 model organisms sorted by family. A) Escherichia coli B) Bacillus subtillis C) 
$463 \quad$ Fig. 4

464 Recovery of novel Transcription Factors in one metagenome and eleven

465 metatranscriptomes. A) PredicTF predicted 792 TFs were predicted in one anaerobic

466 ammonium oxidizing microbial communities from anammox membrane bioreactor

467 (LAC_MetaG_1) and were grouped by family. B) Using 792 TFs predicted in one

468 metagenome, we mapped these TFs for 11 metatranscriptomes of reference from the

469 same bioreactor where the metagenome was recovered.

470

471

472

473

474

475

476

477

478

479

480

481

482

483

484

485

486

487

488 
489 Additional files

490 Additional file 1: Fig. S1

491 Transcription factor (TF) families predicted for Pseudomonas aeruginosa PAO1

492 genome (accession number NC_002516.2) [18] using PredicTF and their mapping to $P$.

493 aeruginosa PAO1 growing in LB medium. A) A total of 199 TFs distributed in 25 TF

494 families were predicted in the P. aeruginosa PAO1 genome. B) These 199 TFs were

495 mapped in the transcriptomic data of a reference of $P$. aeruginosa PAO1 (Bioproject identifier PRJNA479711) [18]. Initially, the mapping was done in the transcriptome of P. aeruginosa PAO1 cultured in LB media. Using this strategy, we were able to map 69 of the 199 predicted TFs to the transcriptome.

499

$500 \quad$ Additional file 2: Fig. S2

501 Transcription Factor (TF) family profiles in three Pseudomonas aeruginosa PAO1 mutants. After the prediction of Transcription Factors (TFs) using P. aeruginosa PAO1 genome, we mapped transcriptomes from three P. aeruginosa PAO1 mutants (Y82,

504 Y71, Y89) cultured in LB media (A, C and F). After, the mapping was done for each $P$. aeruginosa PAO1 mutant cultured in presence of antibiotic cocktail (B, D and E). $P$. aeruginosa PAO1 mutant Y82 (A, B); P. aeruginosa PAO1 mutant Y71 (C, D); P. aeruginosa PAO1 mutant Y89 (E, F).

508

Additional file 3: Table S1

510 Accession number for 5 model organisms, Pseudomonas aeruginosa PAO1 genome and transcriptomes and Complex Microbial Communities used to validate and test PredicTF. 


\section{Additional file 4: Table S2}

516 Transcription factors from the metagenome of an anaerobic ammonium oxidizing

517 microbial community from an anammox membrane bioreactor (LAC_MetaG_1) mined

518 and hand curated from a general annotation generated using Prokka.

519

520 Additional file 5: Table S3

521 Number of Transcription Factors (TFs) per TF family mapped to each of the 11

metatranscriptomes of reference from the same bioreactor where the metagenome

523 (accession number PRJNA511011, NCBI) used to predict the putative TFs was

524 collected. The different metatranscriptomes are represented by their European

525 Nucleotide Archive accession numbers.

526

527

Additional file 6: Fig. S4

528

Bacterial Transcription Factor Data Base (BacTFDB) were created from from two publicly available databases. We collect 390 TFs from CollecTF and 21.581 from UniProt (accessed 8-Sep-2019) accumulating 21.581 Transcription Factor (TF) amino acid sequences. We merged the data from CollecTF and UniProt databases resulting in a total of 21.971 TFs amino acid. We removed redundant TF entries and since PredicTF was designed to also assign TF family, TF sequences lacking a TF family were removed. Finally, a manual inspection was performed to remove misleading of spelling, case sensitive and presence of characters associate to the database header. The final database (BacTFDB) contains a total of 11.691 TF unique sequences.

Additional file 7: Table S4

539 Description of the bacterial transcriptional factors database (BacTFDB) subsets used to

540 train models to predict Trancription Factors in model organisms 


\section{$541 \quad$ Additional file 8}

542 Equations used to calculate PredicTF's accuracy and performance.

543

544

545

546

547

548

549

550

551

552

553

554

555

556

557

558

559

560

561

562

563

564

565

566 


\section{References}

568 1. Liu J, Meng Z, Liu X, Zhang XH. Microbial assembly, interaction, functioning,

569 activity and diversification: a review derived from community compositional data. Mar

$570 \quad$ Life Sci Technol. 2019;1:112-28.

571 2. Browning DF, Busby SJW. The regulation of bacterial transcription initiation. Nat

572 Rev Microbiol. 2004;2:57-65. Nature Publishing Group.

573 3. Browning DF, Butala M, Busby SJW. Bacterial Transcription Factors: Regulation by

574 Pick “N” Mix. J. Mol. Biol. Academic Press. 2019;4067-77.

575 4. Browning DF, Busby SJW. Local and global regulation of transcription initiation in

576 bacteria. Nat Rev Microbiol. 2016;14:638-50. Nature Publishing Group.

577 5. Browning DF, Grainger DC, Busby SJ. Effects of nucleoid-associated proteins on

578 bacterial chromosome structure and gene expression. Curr Opin Microbiol.

579 2010;13:773-80.

580 6. Gama-Castro S, Salgado H, Santos-Zavaleta A, Ledezma-Tejeida D, Muñiz-Rascado

581 L, García-Sotelo JS, et al. RegulonDB version 9.0: high-level integration of gene

582 regulation, coexpression, motif clustering and beyond. Nucleic Acids Res.

583 2016;44:D133-43.

584 7. Sierro N, Makita Y, De hoon M, Nakai K. DBTBS: A database of transcriptional

585 regulation in Bacillus subtilis containing upstream intergenic conservation information.

586 Nucleic Acids Res. 2008;36.

587 8. Gelbart, W. M., Crosby, M., Matthews, B., Rindone, W. P., Chillemi, J., Twombly,

588 S. R, et al. FlyBase: a Drosophila database. Flybase Consortium. Nucleic Acids Res.

589 1998;26:85-8.

590 9. Park J, Park J, Jang S, Kim S, Kong S, Choi J, et al. FTFD: An informatics pipeline 591 supporting phylogenomic analysis of fungal transcription factors. Bioinformatics.

592 2008;24:1024-5.

593 10. Kummerfeld SK. DBD: a transcription factor prediction database. Nucleic Acids 594 Res. 2006;34:D74-81.

595 11. Widder S, Allen RJ, Pfeiffer T, Curtis TP, Wiuf C, Sloan WT, et al. Challenges in 596 microbial ecology: Building predictive understanding of community function and 597 dynamics. ISME J. Springer Nature. 2016;2557-68. Nature Publishing Group.

598 12. Alipanahi B, Delong A, Weirauch MT, Frey BJ. Predicting the sequence 599 specificities of DNA- and RNA-binding proteins by deep learning. Nat Biotechnol. 600 2015;33:831-8. Nature Publishing Group.

601 13. Pan X, Shen H Bin. RNA-protein binding motifs mining with a new hybrid deep

602 learning based cross-domain knowledge integration approach. BMC Bioinformatics.

603 BioMed Central Ltd. 2017;18.

604 14. Kiliç S, White ER, Sagitova DM, Cornish JP, Erill I. CollecTF: A database of 
605 experimentally validated transcription factor-binding sites in Bacteria. Nucleic Acids

606 Res. 2014;42.

607 15. UniProt Consortium T. UniProt: the universal protein knowledgebase. Nucleic

608 Acids Res. 2018;46:2699-2699.

609 16. Hwang W, Yoon SS. Virulence Characteristics and an Action Mode of Antibiotic

610 Resistance in Multidrug-Resistant Pseudomonas aeruginosa. Sci Rep. 2019;9.

611 17. Keren R, Lawrence JE, Zhuang W, Jenkins D, Banfield JF, Alvarez-Cohen L, et al.

612 Increased replication of dissimilatory nitrate-reducing bacteria leads to decreased

613 anammox bioreactor performance. Microbiome. 2020;8. BioMed Central Ltd.

614 18. Seemann T. Prokka: Rapid prokaryotic genome annotation. Bioinformatics.

615 2014;30:2068-9. Oxford University Press.

616 19. Parks DH, Rinke C, Chuvochina M, Chaumeil PA, Woodcroft BJ, Evans PN, et al.

617 Recovery of nearly 8,000 metagenome-assembled genomes substantially expands the

618 tree of life. Nat Microbiol. 2017;2:1533-42. Nature Publishing Group.

619 20. Pasolli E, Asnicar F, Manara S, Zolfo M, Karcher N, Armanini F, et al. Extensive

620 Unexplored Human Microbiome Diversity Revealed by Over 150,000 Genomes from

621 Metagenomes Spanning Age, Geography, and Lifestyle. Cell. 2019;176:649-662.e20.

622 21. Tully BJ, Graham ED, Heidelberg JF. The reconstruction of 2,631 draft

623 metagenome-assembled genomes from the global oceans. Sci Data. 2018;5.

624 22. Arango-Argoty G, Garner E, Pruden A, Heath LS, Vikesland P, Zhang L.

625 DeepARG: A deep learning approach for predicting antibiotic resistance genes from

626 metagenomic data. Microbiome. 2018;6. BioMed Central Ltd.

627 23. Sørensen L, Loog M, Lo P, Ashraf H, Dirksen A, Duin RPW, et al. Image

628 dissimilarity-based quantification of lung disease from CT. Lect Notes Comput Sci.

629 2010;37-44.

630 24. Krueger F. Babraham Bioinformatics - Trim Galore!. Version 0.5.0. 2018. Available

631 from: https://www.bioinformatics.babraham.ac.uk/projects/trim_galore/

632 25. Nurk S, Meleshko D, Korobeynikov A, Pevzner PA. MetaSPAdes: A new versatile

633 metagenomic assembler. Genome Res. 2017;27:824-34.

634 26. Madeira F, Park YM, Lee J, Buso N, Gur T, Madhusoodanan N, et al. The EMBL-

635 EBI search and sequence analysis tools APIs in 2019. Nucleic Acids Res.

636 2019;47:W636-41.

637 27. Langmead B, Salzberg SL. Fast gapped-read alignment with Bowtie 2. Nat

638 Methods. 2012;9:357-9.

639 28. Li H, Handsaker B, Wysoker A, Fennell T, Ruan J, Homer N, et al. The Sequence

640 Alignment/Map format and SAMtools. Bioinformatics. 2009;25:2078-9.

641 29. Buchfink B, Xie C, Huson DH. Fast and sensitive protein alignment using

642 DIAMOND. Nat. Methods. 2014;59-60. Nature Publishing Group. 
643 30. van Merriënboer B, Bahdanau D, Dumoulin V, Serdyuk D, Warde-Farley D,

644 Chorowski J, et al. Blocks and Fuel: Frameworks for deep learning. arxiv.org. 2015.

645 31. Pedregosa F, Michel V, Grisel O, Blondel M, Prettenhofer P, Weiss R, et al. Scikit646 learn: Machine Learning in Python. J. Mach. Learn. Res. 2011; 2825-2830.

647 32. Al-Rfou R, Alain G, Almahairi A, Angermueller C, Bahdanau D, et al. Theano: A

648 Python framework for fast computation of mathematical expressions. arXiv. 2016;1605.

649

650 


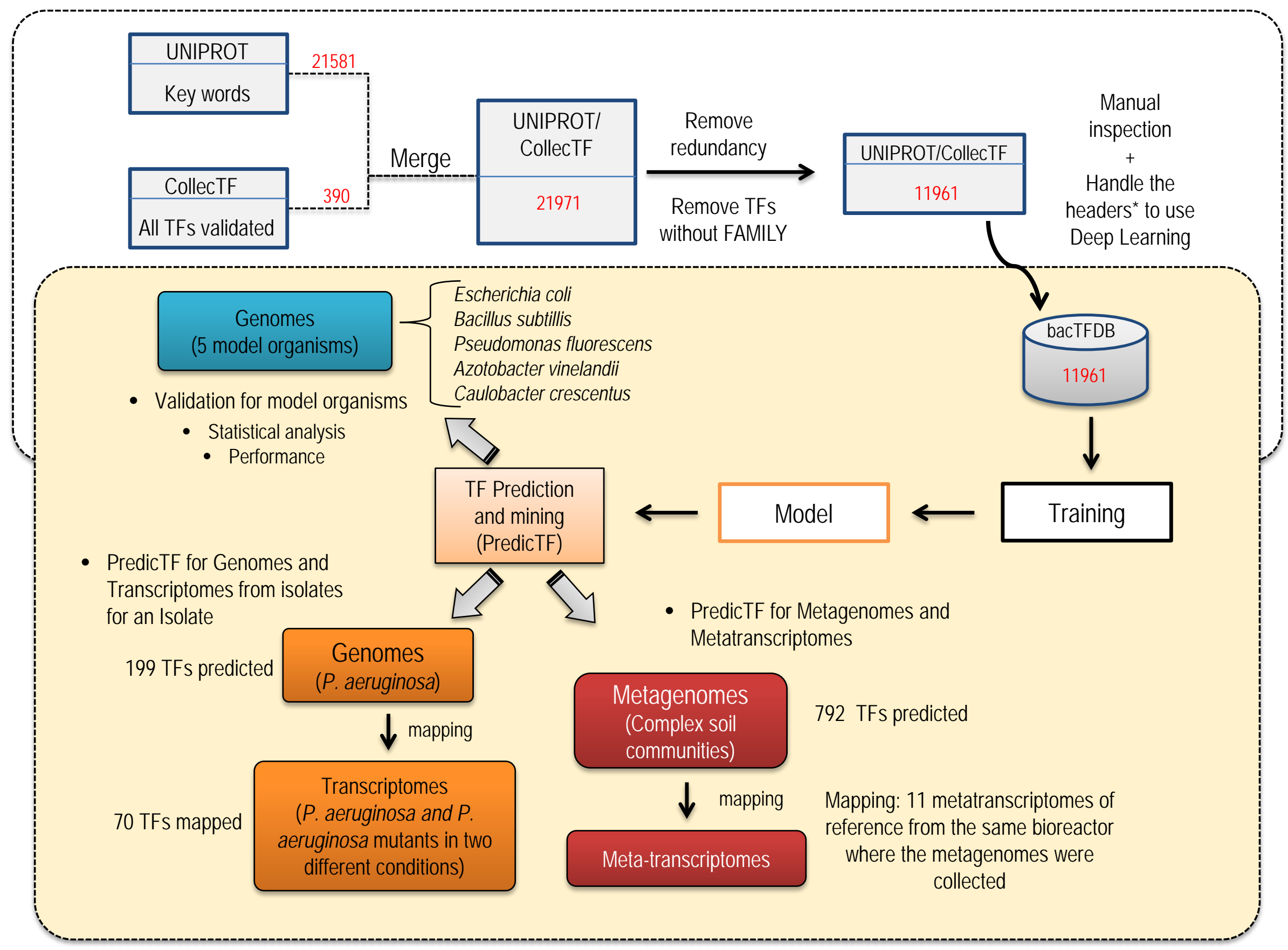


A

\section{Escherichia coli}

AraC/Xyls 》]

IclR ]

Lrp ]

LuxR ]

MarR/SlyA

RpiR o

FNR/CRP

Fur ]

H-NS

Rrf2

XRE $]$

MerR

NarL/FixJ

$\mathrm{NtrC/DctD}$

RelB

$\mathrm{LacI} / \mathrm{GalR}$

TetR

FIS

GntR

OmpR/PhoB

LysR

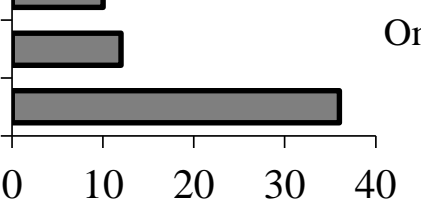

Number of

Transcription Factors
B

Bacillus subtillis

C

Pseudomonas fluorescens

D

Azotobacter vinelandii

ArsR $\square$

CodY $\square$

CsoR

LexA

Lrp

MerR $\square$

CopY

MarR/SlyA

NtrC/DctD

Rrf2

FIS

Fur

LacI/GalR

GntR

NarL/FixJ

OmpR/PhoB

LysR

0

\begin{abstract}
Number of
\end{abstract}
Transcription Factors

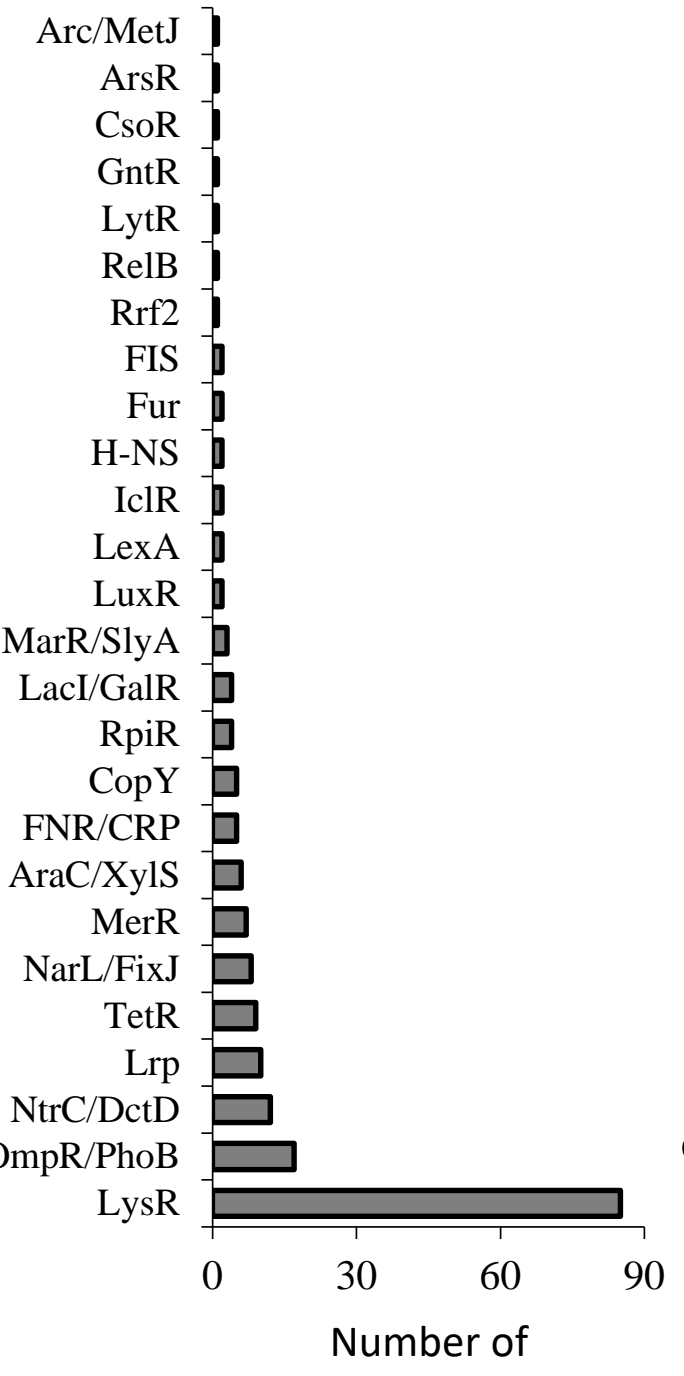

Transcription Factors

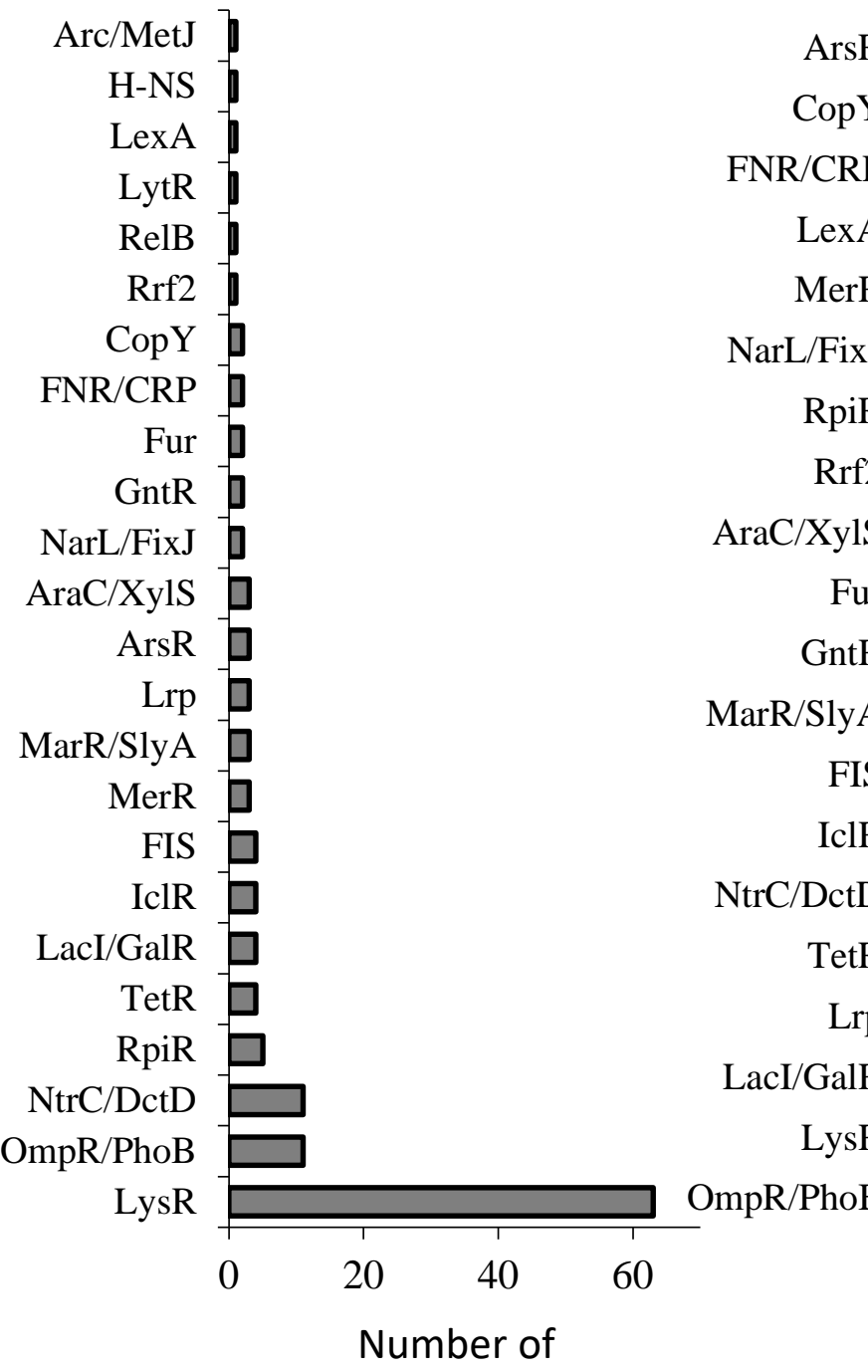

Transcription Factors
E

Caulobacter crescentus 

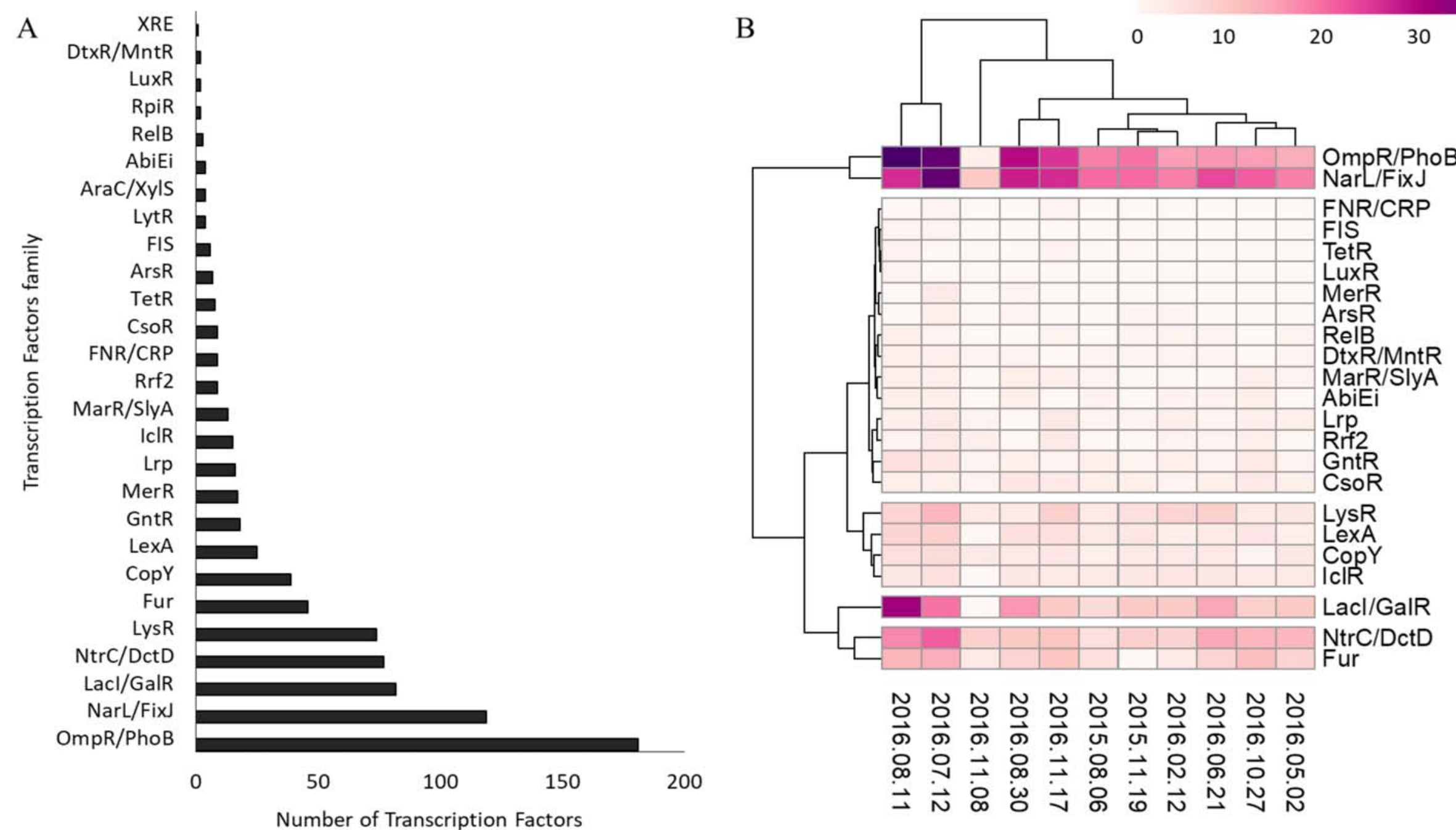

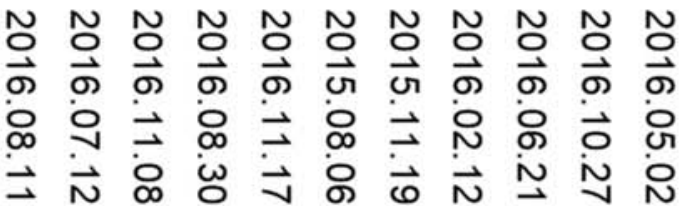

Number of Transcription Factors 